\title{
SPIRAL STRUCTURE IN THE OUTER GALACTIC DISK. I. THE THIRD GALACTIC QUADRANT
}

\author{
Ruben A. VÁzQuez \\ Facultad de Ciencias Astronómicas y Geofísicas de la Universidad Nacional de La Plata, IALP-CONICET, Paseo del Bosque s/n 1900, \\ La Plata, Argentina; rvazquez@fcaglp.fcaglp.unlp.edu.ar \\ JoRge MAY \\ Departamento de Astronomía, Universidad de Chile, Casilla 36-D, Santiago, Chile; jmay@das.uchile.cl \\ Giovanni Carraro ${ }^{1}$ \\ ESO, Alonso de Cordova 3107, Vitacura, Santiago, Chile; gcarraro@eso.org
}

LEONARDO BRONFMAN

Departamento de Astronomía, Universidad de Chile, Casilla 36-D, Santiago, Chile; leo@das.uchile.cl

AndRÉ MoITINHO

SIM/IDL, Faculdade de Ciências da Universidade de Lisboa, Ed. C8, Campo Grande, 1749-016 Lisboa, Portugal; andre@sim.ul.pt

AND

Gustavo Baume

Facultad de Ciencias Astronómicas y Geofísicas de la Universidad Nacional de La Plata, IALP-CONICET, Paseo del Bosque s/n 1900, La Plata, Argentina; gbaume@fcaglp.fcaglp.unlp.edu.ar

Received 2007 April 16; accepted 2007 September 23

\begin{abstract}
We combine optical and radio observations to trace the spiral structure in the third quadrant of the Milky Way. The optical observations consist of a large sample of young open clusters and associations, whereas the radio observations consist of a survey of nearby and distant clouds observed in CO. Both the optical and radio samples are the largest ones thus far presented in the literature. We use this unique material to analyze the behavior of interstellar extinction and to trace the detailed structure of the third Galactic quadrant (TGQ). We find that the outer (Cygnus) grand design spiral arm is traced by stellar and CO components, while the Perseus arm is traced solely by CO and is possibly being disrupted by the crossing of the Local (Orion) arm. The Local arm is traced by CO and young stars toward $l=240^{\circ}$ and extends for over $8 \mathrm{kpc}$ along the line of sight reaching the outer arm. Finally, we characterize the Galactic warp and compare the geometries implied by the young stellar and $\mathrm{CO}$ components.
\end{abstract}

Subject headings: Galaxy: disk - Galaxy: structure - open clusters and associations: general

Online material: color figure

\section{INTRODUCTION}

The complex structure of the Milky Way in the third Galactic quadrant (TGQ) was noticed some time ago with the presence of an unusual velocity field (Brand \& Blitz 1993) and the warp (May et al. 1988; Wouterloot et al. 1990; May et al. 1997) and corrugations that result in the noncoincidence of the $\mathrm{H} \mathrm{I}(21 \mathrm{~cm})$ layer with the formal Galactic plane $\left(b=0^{\circ}\right.$; Burton 1985). Not long ago, Carney \& Seitzer (1993) obtained deep optical colormagnitude diagrams (CMDs) along some lines of sight in the TGQ that allowed the detection of the Galactic warp, identified from sequences of young stars at high negative Galactic latitudes. Recently, Moitinho et al. (2006) produced a map of the young stellar component of the TGQ, which provides a full three-dimensional (3D) view of the warp. Shortly after, Momany et al. (2006) published a detailed analysis of the warp's structure, which the reader is referred to for any additional information.

As for the spiral structure in the TGQ, no clear spatial description had been obtained until very recently by Carraro et al. (2005) and Moitinho et al. (2006). Using stellar (optical) and CO data, they showed for the first time the shapes of the outer arm and a structure extended toward $l \sim 240^{\circ}$, interpreted as being the Local arm, entering the TGQ, confirming a previous suggestion

\footnotetext{
1 On leave from the Dipartimento di Astronomia, Universitá di Padova, Italy.
}

by Moffat et al. (1979). ${ }^{2}$ While earlier CO data (May et al. 1997) could not unveil any clear grand design arm due to limited distance sensitivity, new deeper data (May et al. 2007) actually do show the full outer arm in the TGQ. This has also been very recently found in the $\mathrm{H}$ i study of Levine et al. (2006), who trace spiral features up to $25 \mathrm{kpc}$ from the Galactic center. What remains unclear is the behavior of the Perseus arm, which is very well defined in the second quadrant (e.g., Xu et al. 2006) but hardly detectable in the TGQ.

From what has been delineated above, an interesting aspect emphasized in previous investigations and which deserves a closer analysis is the description of the Local arm in the TGQ. On one hand, from the radio perspective, the strong $\mathrm{CO}$ emission toward Vela - known as the Vela Molecular Ridge - appears to indicate that the Local arm enters the TGQ and extends in the direction of the Vela region $\left(255^{\circ}-275^{\circ}\right.$; May et al. 1988; Murphy $\&$ May 1991) up to the limit of the CO survey at $8 \mathrm{kpc}$ from the Sun. Such evidence is also present in the $\mathrm{H}$ I study performed by Burton (1985). On the other hand, optical stellar data reveal the existence of an elongated structure toward $l \sim 240^{\circ}$ reaching the outer arm (Moitinho et al. 2006). This latter structure is also traced

\footnotetext{
2 Strictly speaking, the "outer arm" mentioned by Moffat et al. (1979) is closer than what is found in these studies.
} 
TABLE 1

Basic Parameters of the New Clusters and Background Populations

\begin{tabular}{|c|c|c|c|c|c|c|c|c|c|c|}
\hline Field & Name & $\begin{array}{c}l \\
(\mathrm{deg})\end{array}$ & $\begin{array}{c}b \\
(\mathrm{deg})\end{array}$ & $\begin{array}{c}(m-M) \\
(\mathrm{mag})\end{array}$ & $\begin{array}{c}E(B-V) \\
(\mathrm{mag})\end{array}$ & $\begin{array}{c}d_{\odot} \\
(\mathrm{kpc})\end{array}$ & $\begin{array}{l}\text { Age } \\
(\mathrm{Myr})\end{array}$ & $\begin{array}{c}X \\
(\mathrm{kpc})\end{array}$ & $\begin{array}{c}Y \\
(\mathrm{kpc})\end{array}$ & $\begin{array}{c}Z \\
(\mathrm{kpc})\end{array}$ \\
\hline \multirow[t]{2}{*}{$1 \ldots \ldots \ldots \ldots$} & Ruprecht 1 & 223.990 & -9.690 & $12.00 \pm 0.30$ & $0.30 \pm 0.10$ & $1.60 \pm 0.20$ & $300 \pm 50$ & -0.958 & 0.993 & -0.236 \\
\hline & BP Ruprecht 1 & 223.990 & -9.690 & $15.85 \pm 0.35$ & $0.40 \pm 0.15$ & $8.40 \pm 0.70$ & $\leq 100$ & -5.751 & 5.957 & -1.414 \\
\hline $2 \ldots$ & vdB-Hagen $92^{a}$ & 224.570 & -2.490 & $10.40 \pm 0.15$ & $0.15 \pm 0.05$ & $0.97 \pm 0.07$ & $40 \pm 10$ & -0.680 & 0.690 & -0.042 \\
\hline \multirow[t]{2}{*}{$3 \ldots \ldots \ldots \ldots \ldots$} & Berkeley 76 & 225.099 & -1.998 & $16.50 \pm 0.40$ & $0.40 \pm 0.15$ & $11.30 \pm 1.50$ & $2000 \pm 250$ & -7.645 & 7.619 & -0.377 \\
\hline & BP Berkeley 76 & 225.099 & -1.998 & $15.80 \pm 0.50$ & $0.50 \pm 0.02$ & $7.30 \pm 1.60$ & $\leq 100$ & -5.168 & 5.150 & -0.255 \\
\hline \multirow[t]{2}{*}{$4 \ldots \ldots \ldots \ldots \ldots$} & Haffner 4 & 227.900 & -3.586 & $14.75 \pm 0.20$ & $0.53 \pm 0.07$ & $4.20 \pm 0.50$ & $300 \pm 50$ & -2.740 & 2.476 & -0.231 \\
\hline & BP Haffner 4 & 227.900 & -3.586 & $15.85 \pm 0.60$ & $0.50 \pm 0.07$ & $7.60 \pm 1.20$ & $\leq 100$ & -5.628 & 5.085 & -0.475 \\
\hline \multirow[t]{2}{*}{$5 \ldots \ldots \ldots \ldots \ldots$} & Auner 1 & 232.110 & -6.200 & $15.75 \pm 0.15$ & $0.32 \pm 0.05$ & $8.90 \pm 0.60$ & $3250 \pm 250$ & -6.800 & 5.300 & -0.990 \\
\hline & BP Auner 1 & 232.110 & -6.200 & $16.00 \pm 0.30$ & $0.40 \pm 0.10$ & $8.95 \pm 0.40$ & $\leq 100$ & -7.022 & 5.464 & -0.967 \\
\hline \multirow[t]{2}{*}{$6 \ldots \ldots \ldots \ldots \ldots$} & Ruprecht 10 & 232.553 & -5.584 & $12.80 \pm 0.40$ & $0.25 \pm 0.15$ & $2.50 \pm 0.40$ & $800 \pm 150$ & -1.738 & 1.331 & -0.214 \\
\hline & BP Ruprecht 10 & 232.553 & -5.584 & $15.95 \pm 0.50$ & $0.50 \pm 0.10$ & $7.90 \pm 1.80$ & $\leq 100$ & -6.242 & 4.781 & -0.769 \\
\hline $7 \ldots \ldots$ & BP Ruprecht 150 & 240.010 & -9.647 & $15.70 \pm 0.50$ & $0.35 \pm 0.05$ & $8.60 \pm 1.80$ & $\leq 100$ & -7.343 & 4.238 & -1.441 \\
\hline $8 \ldots \ldots \ldots \ldots \ldots$ & Haffner 11 & 242.395 & -3.544 & $15.25 \pm 0.25$ & $0.50 \pm 0.10$ & $5.50 \pm 0.50$ & $800 \pm 100$ & -5.307 & 2.775 & -0.371 \\
\hline \multirow[t]{2}{*}{$9 \ldots \ldots \ldots \ldots$} & Haffner 7 & 242.673 & -6.804 & $14.55 \pm 0.25$ & $0.27 \pm 0.15$ & $5.50 \pm 0.50$ & $600 \pm 100$ & -3.705 & 1.915 & -0.498 \\
\hline & BP Haffner 7 & 242.673 & -6.804 & $15.75 \pm 0.85$ & $0.20 \pm 0.10$ & $10.60 \pm 3.00$ & $\leq 100$ & -9.351 & 4.832 & -1.256 \\
\hline $10 \ldots \ldots \ldots \ldots \ldots$ & BP CMa & 244.000 & -8.000 & $16.04 \pm 0.10$ & $0.40 \pm 0.10$ & $9.30 \pm 0.40$ & $\leq 100$ & -8.100 & 3.900 & -1.300 \\
\hline \multirow[t]{3}{*}{$11 \ldots \ldots \ldots \ldots \ldots$} & Ruprecht $30^{\mathrm{a}}$ & 246.419 & -4.046 & $12.10 \pm 0.20$ & $0.47 \pm 0.03$ & $1.30 \pm 0.20$ & $60 \pm 15$ & -1.188 & 0.519 & -0.092 \\
\hline & SC Ruprecht 30 & 246.289 & -4.030 & $17.25 \pm 0.10$ & $0.55 \pm 0.05$ & $12.60 \pm 0.40$ & $20 \pm 5$ & -11.508 & 5.054 & -0.886 \\
\hline & BP Ruprecht 30 & 246.419 & -4.046 & $16.65 \pm 0.15$ & $0.55 \pm 0.05$ & $9.85 \pm 1.30$ & $\leq 100$ & -9.005 & 3.931 & -0.695 \\
\hline $12 \ldots \ldots \ldots \ldots \ldots$ & Haffner 15 & 247.952 & -4.158 & $14.20 \pm 0.20$ & $0.70 \pm 0.15$ & $2.50 \pm 0.30$ & $60 \overline{0} \pm 150$ & -2.219 & 0.899 & -0.174 \\
\hline
\end{tabular}

${ }^{\text {a }}$ Very preliminary values for distance, reddening and age. Object seems to be just a sparse group of young and midage stars.

by $\mathrm{CO}$ clouds and similarly appears to be the extension of the Local arm in the TGQ.

In Moitinho et al. (2006) we have presented a 3D map of the TGQ, built from a catalog of young open clusters and fields containing early-type stars, complemented with $\mathrm{CO}$ observations of distant clouds. The focus in the Moitinho et al. (2006) Letter was on showing how observational evidence interpreted as a galaxy being accreted by the Milky Way (the Canis Major dwarf galaxy; Bellazzini et al. 2004; Martin et al. 2004; Martínez-Delgado et al. 2005) was in fact more naturally explained by the previously unclear broad spiral structure, together with the warp, in the TGQ (Momany et al. 2004). In this paper, we add new stellar fields and $\mathrm{CO}$ data to produce an updated version of the map from Moitinho et al. (2006). In contrast with the Letter, we now focus on a detailed analysis of all the detected spiral features and the warp in the TGQ. In addition, data analysis and the reddening pattern of the TGQ are discussed in depth, which was not done in the previous work.

The remainder of the paper is organized as follows. In $\S 2$ we present and discuss the optical sample, illustrating the details of the derivation of fundamental parameters. Section 3 describes the acquisition process, velocity resolution, and sensitivity of the $\mathrm{CO}$ molecular data sample used in this paper. The effect of reddening and its variation across the TGQ is analyzed in $\S 4$. Our analysis of the derived Galactic structure and the warp signatures are given in $\S \S 5$ and 6, respectively. Section 7 includes the final remarks and the conclusions.

\section{OPTICAL SAMPLE}

The sample in this study is an extension of the one used in Moitinho et al. (2006). With respect to that study we are adding 12 fields, where we can recognize star clusters and/or young background populations (blue plumes [BPs]) similar to the ones described in Carraro et al. (2005) that are used for tracing spiral features.

\subsection{The Data}

This sample extension comes from an observing run conducted in Cerro Tololo with the $1.0 \mathrm{~m}$ telescope on nights 2005
November 28 -December 4 . The telescope is operated by the SMARTS consortium ${ }^{3}$ and hosts a new $4 \mathrm{k} \times 4 \mathrm{k}$ CCD camera with a pixel scale of $0.289^{\prime \prime}$ pixel $^{-1}$, which allows us to cover a field of $20^{\prime} \times 20^{\prime}$ on the sky. All nights were photometric with an average seeing of $0.8^{\prime \prime}-1.3^{\prime \prime}$.

$U B V I$ photometry has been obtained using the $\mathrm{IRAF}^{4}$ packages CCDRED, DAOPHOT, and PHOTCAL following the pointspread function (PSF) method (Stetson 1987). Calibration was secured through observations of several (Landolt 1992) standard fields.

Since all the nights were photometric, a global photometric solution has been derived using all the standard stars' (about 200) observations. The typical rms of the zero points are $0.04,0.03$, 0.03 , and 0.03 in $U, B, V$, and $I$, respectively. A detailed description of the data reduction will be presented in forthcoming papers (e.g., G. Carraro et al. 2008, in preparation), where all the clusters here presented will be discussed closely.

\subsection{Fundamental Parameters}

Below we describe the procedures followed in deriving the fundamental parameters - reddening, distance, and age - of the stellar populations found in the 12 fields listed in Table 1. This paper is concerned with the spiral structure; therefore, from the sample in Table 1 we only selected stellar groups younger than $100 \mathrm{Myr}$, since older groups are not useful as spiral structure tracers. Therefore, we are not using Ruprecht 1, Ruprecht 10, Haffner 11, Haffner 4, Haffner 15, Haffner 7, Berkeley 76, or Auner 1. In some cases (Ruprecht 150 and the field centered in the Canis Major overdensity) we do not find any star cluster but only a diffuse young population. Photometric diagrams of all these fields are included in a forthcoming article (G. Carraro et al. 2008, in preparation) but for Auner 1, which is presented in Carraro et al. (2007). Nevertheless, the old clusters of our sample are used together with the young ones to describe the reddening pattern.

\footnotetext{
${ }^{3}$ See http://www.astro.yale.edu/smarts/.

4 IRAF is distributed by NOAO, which is operated by AURA, Inc., under cooperative agreement with the NSF.
} 
According to the age and the places where they are located, clusters present a variety of challenges in deriving their intrinsic parameters. In the case of very young clusters, they are normally close to the Galactic plane where interstellar absorption and crowded stellar fields make membership assignments of faint stars difficult. Yet they contain massive (i.e., hot) stars that can be used to derive their parameters. The classical photometric method to address memberships in young clusters (e.g., Baume et al. 1999) simultaneously checks the consistency of the location of each star in several photometric diagrams (two-color diagrams [TCDs] and different CMDs). This method works well for stars with spectral types earlier than A0 while becoming almost impractical to segregate late faint cluster members from field stars in crowded fields. It has been criticized by Abt (1979), but as stated by Turner et al. (1980) and later emphasized by Forbes (1996), it is an efficient tool when membership estimates rely on a careful inspection of the TCD and consistent reddening solutions are applied. This is a crucial point, as age determinations of young clusters may vary up to $100 \%$ for clusters younger than $10^{7} \mathrm{yr}$ just because of a poor reddening determination and/or bad membership assignments (since both affect the identification of the turnoff point position). Even in the bright portion of the CMDs contamination by field stars may exist. However, since the $U-B$ index is more sensitive to stellar temperature changes than $B-V$ (Meynet et al. 1993), the $V$ versus $U-B$ diagram in young clusters is quite useful for eliminating even most $\mathrm{A}-\mathrm{F}$ type foreground stars that tend to contaminate the vertical cluster sequence in the $V / B-V$ plane. The outcome is that foreground (colder stars in the mean) separate from hot cluster members. Further membership control was always done if MK spectral types were available.

Accurate distances require identifying clear sequences along a broad range of magnitudes. To reduce field star contamination in the lower main sequence we restrict the analysis to stars within the cluster limits. The limits are determined by inspection of radial density profiles created from star counts (e.g., Carraro et al. 2007) and set at the distance where cluster stars reach the same density numbers as field stars. This approach will normally be enough to produce clear cluster sequences, thus allowing straightforward distance determinations. As a final remark, the bright (massive) stars often include fast rotators, Be-type stars, and binaries (just to mention some effects) that widen the cluster sequence and lead to poorly constrained cluster parameters. However, the number of these stars and the intensity of the phenomena are known to vary from cluster to cluster. Some are well known for the profusion of peculiar stars (e.g., Be stars in NGC 3766; Moitinho et al. 1997), but in most cases their numbers will be small enough so that they will stand out from the rest of the cluster sequence and will not affect the derived parameters, as is the case of the sample presented here.

Mean color excesses, $E_{(B-V)}$ and $E_{(U-B)}$, were determined by shifting the Schmidt-Kaler (1982) zero-age main sequence (ZAMS) along the reddening line [given by the standard relation $E_{(U-B)}=$ $\left.0.72 E_{(B-V)}+0.05 E_{(B-V)}^{2}\right]$ until the best fit to blue cluster members was achieved. Errors in the mean color excess as listed in Table 1 were estimated by eye.

To compute cluster distances we must remove the effect of interstellar absorption, $A_{V}$. This requires the knowledge of the ratio of total to selective absorption, $R=A_{V} / E_{(B-V)}$, which can be higher than the normal value $R=3.1$ in regions of recent star formation. Distances and ages of the youngest clusters could then be significantly biased by wrongly assuming the standard value. Although the analysis in Moitinho (2001) of the fields of several open clusters in the TGQ suggests that the extinction law in the TGQ is normal, an independent check of $R$ was performed: For every cluster field, the stellar distribution in the $B-V / V-I$ plane, where the slope of the reddening vector for normal absorbing material is given by $E_{(V-I)} / E_{(B-V)}=1.244$ (Dean et al. 1978 ), was carefully examined. Since in this diagram the slope of the stellar distribution and the standard reddening line are very similar, deviations between them are indicative of a nonstandard reddening law. Cluster distances were derived by superposing the Schmidt-Kaler (1982) ZAMS to the reddening- and absorptioncorrected CMDs. The fitting error to each cluster was estimated by eye.

Cluster ages have been derived by superposing the solar metallicity isochrones from Girardi et al. (2000) onto the reddening-free sequences, as usual for star clusters. Emphasis was put in achieving the best fit for all the members located in the upper sequence. The age errors listed in Table 1 are more lower and upper limits. There are, however, cases in which the fitting is ambiguous so that more than one isochrone was needed to trace the cluster sequence. This fact is assumed to be caused either by binarity or by the remaining material of the star formation process (only in cases of extremely young clusters).

\section{MOLECULAR CLOUD SAMPLE IN THE THIRD GALACTIC QUADRANT}

CO surveys of the molecular cloud distribution in the TGQ of the Galaxy have been carried out by May et al. (1997) for $d \geq$ $2 \mathrm{kpc}$, and more recently by May et al. (2007) for $R \geq 14.5 \mathrm{kpc}$. The former data set was obtained completely with the CfA-Chile $1.2 \mathrm{~m}$ millimeter-wave telescope at Cerro Tololo; the latter one includes observations at higher resolution and sensitivity obtained with both the CfA-Chile telescope and the NANTEN $4 \mathrm{~m}$ telescope, from the University of Nagoya, then at Cerro Las Campanas. The CfA-Chile telescope is a $1.2 \mathrm{~m}$ Cassegrain with a beam width (FWHM) of $8.8^{\prime}$ at $115 \mathrm{GHz}$, the frequency of the $\mathrm{CO}$ $(J=1-0)$ transition and a main beam efficiency of 0.82 (Cohen 1983; Bronfman et al. 1988); the NANTEN is a single-dish $4 \mathrm{~m}$ telescope with a beam width of $2.6^{\prime}$ at $115 \mathrm{GHz}$, and a mainbeam efficiency of 0.89 (Ogawa et al. 1991).

The survey of May et al. (1997) presented a list of 177 molecular clouds mapped in $\mathrm{CO}$, with the CfA-Chile telescope, at a sampling of $0.25^{\circ}$ for $R \leq 12 \mathrm{kpc}$ and of $0.125^{\circ}$ for $12 \mathrm{kpc} \leq$ $R \leq 14.5 \mathrm{kpc}$. The rms noise temperature of the spectra, in $T_{a}$ units, is better than $0.1 \mathrm{~K}$ at a velocity resolution of $1.3 \mathrm{~km} \mathrm{~s}^{-1}$. The new catalog of May et al. (2007) presents 46 molecular clouds with distances beyond $14.5 \mathrm{kpc}$ from the Galactic center. These distant clouds were mapped, with the CfA-Chile telescope, at a sampling interval of $3.75^{\prime}$ (full sampling). The velocity resolution of the spectra was improved to $0.26 \mathrm{~km} \mathrm{~s}^{-1}$, and spectra were integrated to obtain an rms noise temperature per channel, in $T_{a}$ units, better than $0.18 \mathrm{~K}$, corresponding to $0.08 \mathrm{~K}$ when smoothed to a velocity resolution of $1.3 \mathrm{~km} \mathrm{~s}^{-1}$. The overall sensitivity of the new maps, when smoothed to $0.125^{\circ}$, is therefore 2.5 times better than that for the May et al. (1997) maps.

The catalog of 46 clouds mapped in CO by May et al. (2007) contains 14 sources that were either unresolved by the CfA-Chile telescope beam or whose detection was dubious because of poor signal-to-noise ratio. These 14 sources were then mapped with the NANTEN telescope, confirming their detection and obtaining their physical parameters. The NANTEN observations were carried out with a sampling interval of $2.5^{\prime}$, a typical rms, in $T_{a}$ units, of $0.17 \mathrm{~K}$, and a velocity resolution of $0.1 \mathrm{~km} \mathrm{~s}^{-1}$. Therefore, the spatial resolution of the NANTEN maps is 3 times better than the CfA-Chile maps, with about the same sensitivity per beam at a velocity resolution smoothed to $0.26 \mathrm{~km} \mathrm{~s}^{-1}$. A detail of the main parameters of the CO surveys can be found in Table 2. Since 
TABLE 2

Main Parameters of CO Surveys

\begin{tabular}{|c|c|c|c|c|}
\hline \multirow{2}{*}{$\begin{array}{c}\text { Parameter } \\
\text { Telescope } \ldots \ldots \ldots \ldots \ldots \ldots \ldots \ldots \ldots \ldots \ldots\end{array}$} & \multicolumn{2}{|c|}{ May et al. (1997) } & \multicolumn{2}{|c|}{ May et al. (2007) } \\
\hline & CfA-Chile & CfA-Chile & CfA-Chile & Nanten \\
\hline Diameter.... & $1.2 \mathrm{~m}$ & $1.2 \mathrm{~m}$ & $1.2 \mathrm{~m}$ & $4 \mathrm{~m}$ \\
\hline Telescope HPBW ${ }^{\mathrm{a}}$. & $8.8^{\prime}$ & $8.8^{\prime}$ & $8.8^{\prime}$ & $2.6^{\prime}$ \\
\hline Sampling . & $15^{\prime}$ & $7.5^{\prime}$ & $3.75^{\prime}$ & $2.5^{\prime}$ \\
\hline Velocity resolution $\left(\mathrm{km} \mathrm{s}^{-1}\right) \ldots \ldots$ & 1.3 & 1.3 & 0.26 & 0.1 \\
\hline rms sensitivity $\left(\sigma\left[T_{A}^{*}\right]\right)^{\mathrm{b}} \ldots \ldots \ldots \ldots \ldots \ldots$ & $\leq 0.10 \mathrm{~K}$ & $\leq 0.10 \mathrm{~K}$ & $\leq 0.08 \mathrm{~K}$ & $\leq 0.07$ \\
\hline Number of clouds ............................. & 85 & 92 & 32 & 14 \\
\hline Distance $(\mathrm{kpc}) \ldots \ldots \ldots \ldots \ldots \ldots \ldots \ldots \ldots \ldots \ldots \ldots \ldots$ & $d>2 ; R \leq 12$ & $R>12$ & $R>14.5$ & $R>14.5$ \\
\hline
\end{tabular}

${ }^{\text {a }}$ At $115 \mathrm{GHz}$

${ }^{\mathrm{b}}$ At velocity resolution of $1.3 \mathrm{~km} \mathrm{~s}^{-1}$.

outside the solar circle there is no distance ambiguity, heliocentric distances for all $\mathrm{CO}$ clouds in Table 3 (which gives the basic $\mathrm{CO}$ clouds parameters) have been determined kinematically, using the rotation curve of Brand \& Blitz (1993) with the galactic constants $\Theta_{\odot}=220 \mathrm{~km} \mathrm{~s}^{-1}$ and $R_{\odot}=8.5 \mathrm{kpc}$.

\section{THE THIRD GALACTIC QUADRANT: THE REDDENING PATTERN}

Figure 1 shows the plots of individual reddening values of all clusters in our sample against Galactic longitude, latitude, and distance to the Sun. We use different symbols to identify young clusters ( filled circles), BPs (open circles), and the rest of the clusters (starred symbols). The top left panel in Figure $1\left[E_{(B-V)}\right.$ vs. Galactic longitude] shows just a few clusters at $180^{\circ}<l<210^{\circ}$, including the young one NGC 2129 (Carraro et al. 2006) and other older objects, with high reddening (from 0.4 to $0.8-0.9$ $\mathrm{mag}$ ); the bottom left panel (distance from the Sun against Galactic longitude) shows that they all are nearby clusters at no more than $\approx 4 \mathrm{kpc}$. It seems that the reddening increases strongly beyond $4 \mathrm{kpc}$, and for this reason, farther clusters (if any) or other optical arm tracers are not easily detected in this part of the Galaxy. An absorption window then opens in the top left panel at $215^{\circ}<l<255^{\circ}$ within which we find reddening variations from approximately 0.1 to $1 \mathrm{mag}$, reaching high values, above $1.2 \mathrm{mag}$ (S305 and S309) at $235^{\circ}$, but with a mean of $0.4-0.5$ mag along most lines of sight. We remark on the paucity in young cluster distances in the top left panel: there is a nearby group of young clusters with distances up to $3 \mathrm{kpc}$, many of them falling well below the Galactic plane (Fig. 1, bottom right panel). Then a second more populated group of young clusters and BPs extending up to $12 \mathrm{kpc}$ appears. While the separation between these two groups is about $6 \mathrm{kpc}$ at $l=215^{\circ}$, it decreases with increasing longitude down to a minimum at $l=240^{\circ}$. This paucity is somehow mostly occupied by old clusters showing a high dispersion around the formal Galactic plane at $b=0^{\circ}$ as seen in the bottom right panel. Beyond $\approx 255^{\circ}$, the situation changes again: few young clusters remain in our sample, some of them show high reddening values, up to $0.8 \mathrm{mag}$ (e.g., Pismis 8 and 13; Giorgi et al. 2005), while others present reddening lower than $0.3 \mathrm{mag}$. The bottom left panel shows that they are all nearby objects too.

TABLE 3

Basic Parameters of the New Distant CO Clouds

\begin{tabular}{|c|c|c|c|c|c|c|c|c|c|}
\hline $\begin{array}{c}l \\
(\operatorname{deg})\end{array}$ & $\begin{array}{c}b \\
(\mathrm{deg})\end{array}$ & $\begin{array}{c}V_{\mathrm{lsr}} \\
\left(\mathrm{km} \mathrm{s}^{-1}\right)\end{array}$ & $\begin{array}{c}d_{\odot} \\
(\mathrm{kpc})\end{array}$ & $\begin{array}{c}Z \\
(\mathrm{pc})\end{array}$ & $\begin{array}{c}l \\
(\operatorname{deg})\end{array}$ & $\begin{array}{c}b \\
(\mathrm{deg})\end{array}$ & $\begin{array}{c}V_{\mathrm{lsr}} \\
\left(\mathrm{km} \mathrm{s}^{-1}\right)\end{array}$ & $\begin{array}{c}d_{\odot} \\
(\mathrm{kpc})\end{array}$ & $\begin{array}{c}Z \\
(\mathrm{pc})\end{array}$ \\
\hline $195.50 \ldots \ldots \ldots \ldots . .$. & 0.50 & 31.73 & 10.738 & 93.7 & $249.41 \ldots \ldots \ldots \ldots$ & -2.22 & 103.71 & 12.716 & -494.8 \\
\hline $195.68 \ldots \ldots \ldots \ldots$ & -0.12 & 32.44 & 10.990 & -23.9 & $249.61 \ldots \ldots \ldots \ldots$ & -2.10 & 106.08 & 13.195 & -484.6 \\
\hline $195.93 \ldots \ldots \ldots \ldots$ & -0.68 & 32.78 & 10.875 & -130.4 & $250.00 \ldots \ldots \ldots \ldots$ & -3.37 & 98.42 & 11.696 & -689.7 \\
\hline $204.68 \ldots \ldots \ldots \ldots . . .$. & 0.00 & 45.97 & 9.407 & 0.0 & $250.04 \ldots \ldots \ldots \ldots$ & -3.37 & 97.34 & 11.502 & -678.3 \\
\hline $208.37 \ldots \ldots \ldots \ldots . . .$. & -1.81 & 52.48 & 9.604 & -303.8 & $250.12 \ldots \ldots \ldots \ldots$ & -3.12 & 94.85 & 11.065 & 604.0 \\
\hline $208.81 \ldots \ldots \ldots \ldots . .$. & -1.87 & 50.53 & 8.710 & -285.1 & $250.68 \ldots \ldots \ldots \ldots$ & -3.56 & 89.79 & 10.238 & -637.3 \\
\hline $211.06 \ldots \ldots \ldots \ldots . . .$. & 1.18 & 54.72 & 8.990 & 186.3 & $250.93 \ldots \ldots \ldots \ldots$ & -5.12 & 88.80 & 10.088 & -904.7 \\
\hline $215.87 \ldots \ldots \ldots \ldots . .$. & -2.00 & 57.72 & 8.066 & -281.6 & $252.04 \ldots \ldots \ldots \ldots$ & -4.16 & 94.79 & 11.085 & -807.5 \\
\hline $217.62 \ldots \ldots \ldots \ldots$ & -2.62 & 63.86 & 9.068 & -415.7 & $252.08 \ldots \ldots \ldots \ldots$ & -4.04 & 88.89 & 10.138 & -716.3 \\
\hline $218.62 \ldots \ldots \ldots \ldots . .$. & 0.25 & 59.53 & 7.763 & 33.8 & $252.12 \ldots \ldots \ldots \ldots$ & -4.25 & 102.49 & 12.457 & -925.7 \\
\hline $218.75 \ldots \ldots \ldots \ldots$ & -0.43 & 56.56 & 7.110 & -54.2 & $254.62 \ldots \ldots \ldots \ldots$ & -0.93 & 87.49 & 10.039 & -164.2 \\
\hline $221.75 \ldots \ldots \ldots \ldots . .$. & 0.56 & 63.36 & 7.896 & 77.5 & $254.87 \ldots \ldots \ldots \ldots$ & -0.93 & 86.84 & 9.957 & -162.9 \\
\hline $221.75 \ldots \ldots \ldots \ldots . .$. & 0.31 & 63.25 & 7.874 & 42.9 & $256.18 \ldots \ldots \ldots \ldots$ & -1.50 & 88.00 & 10.207 & -267.2 \\
\hline $223.75 \ldots \ldots \ldots \ldots . .$. & -2.00 & 63.04 & 7.473 & -260.9 & $257.56 \ldots \ldots \ldots \ldots$ & -2.18 & 94.70 & 11.323 & -432.5 \\
\hline $226.43 \ldots \ldots \ldots \ldots . .$. & -0.18 & 66.30 & 7.674 & -25.1 & $257.56 \ldots \ldots \ldots \ldots$ & -2.18 & 90.80 & 10.718 & -409.3 \\
\hline $229.75 \ldots \ldots \ldots \ldots$ & 0.06 & 70.44 & 7.992 & 8.7 & $259.16 \ldots \ldots \ldots \ldots$ & -1.33 & 92.89 & 11.159 & -259.7 \\
\hline $235.37 \ldots \ldots \ldots \ldots$ & -1.75 & 81.12 & 9.304 & -284.2 & $263.60 \ldots \ldots \ldots \ldots$ & -3.97 & 111.01 & 14.674 & -1020.7 \\
\hline $235.68 \ldots \ldots \ldots \ldots$ & -1.18 & 75.06 & 8.229 & -170.5 & $263.80 \ldots \ldots \ldots \ldots$ & -4.14 & 114.11 & 15.314 & -1108.4 \\
\hline $237.25 \ldots \ldots \ldots \ldots$ & -1.25 & 77.34 & 8.500 & -185.4 & $263.85 \ldots \ldots \ldots \ldots$ & -4.14 & 111.26 & 14.747 & -1067.4 \\
\hline $237.25 \ldots \ldots \ldots \ldots . .$. & -1.20 & 75.21 & 8.156 & -177.9 & $267.41 \ldots \ldots \ldots \ldots$ & -4.00 & 108.17 & 14.566 & -1018.4 \\
\hline $239.12 \ldots \ldots \ldots \ldots$ & -1.87 & 83.13 & 9.359 & -306.3 & $269.60 \ldots \ldots \ldots \ldots$ & -3.78 & 108.95 & 15.010 & -992.4 \\
\hline $244.43 \ldots \ldots \ldots \ldots$ & -1.93 & 92.73 & 10.783 & -364.7 & $271.05 \ldots \ldots \ldots \ldots$ & -5.51 & 111.44 & 15.700 & -1507.5 \\
\hline $249.18 \ldots \ldots \ldots \ldots . . .$. & -3.81 & 89.67 & 10.193 & -679.2 & $271.45 \ldots \ldots \ldots \ldots$ & -2.63 & 93.83 & 12.762 & -585.5 \\
\hline
\end{tabular}




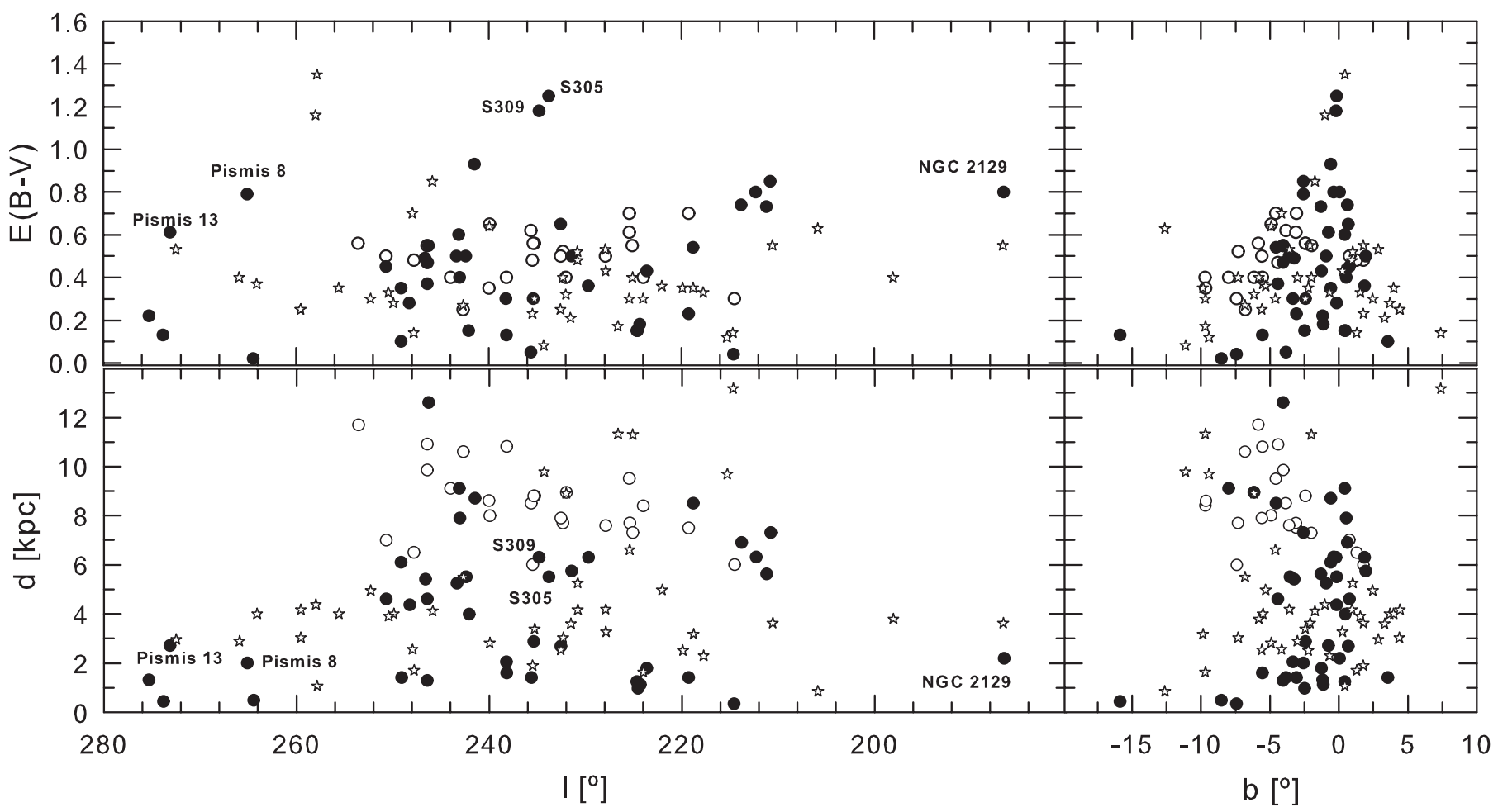

FIG. 1.- Reddening path and distances in the TGQ. Top left and right: $E_{(B-V)}$ vs. Galactic longitude and latitude, respectively. Bottom left and right: Same as top, but for distances from the Sun. Filled and open circles indicate clusters and BPs. Open stars show old clusters. Some objects discussed in the text are indicated.

Something similar happens with old clusters such as Pismis 3 and Saurer 2, which despite being close to the formal galactic plane and the Sun are affected by the highest reddening found in this small sector of the TGQ, above $\sim 1.2$ mag.

In the 1960s, Fitzgerald (1968, hereafter F68) carried out a large-scale reddening distribution analysis using distances and reddening values derived for isolated stars and open cluster stars according to their spectral types and colors. Due to observational limitations at that epoch, the study is mostly restricted to a $2 \mathrm{kpc}$ radius around the Sun. His series of panels 46-59 plot the reddening of the whole TGQ against the distance to the Sun. His Figure 4 sketches the dust cloud distribution projected onto the Galactic plane, while his Figures 5-8 depict the latitude distribution of absorbing clouds at different distances from the Sun. A comparison of F68 findings to ours is therefore pertinent now.

Several features in common between the F68 reddening analysis and ours should be emphasized: we can see distant objects (clusters and BPs) in the region from $215^{\circ}$ to $255^{\circ}$ thanks to the existence of a wide absorption window (the so-called Fitzgerald window) convincingly proved in F68. This window is limited at $215^{\circ}$ by a dense cloud, labeled $\mathrm{K}$ in Figure 4 of F68, that covers from $185^{\circ}$ to $210^{\circ}$ and is followed by two other far regions of medium absorption. The reddening introduced by this region $\mathrm{K}$, placed at less than $1 \mathrm{kpc}$ from the Sun, is over $1.0 \mathrm{mag}$ and spans the range of longitudes where just a few clusters are included in our sample. However, even in this obscured sector, nine $\mathrm{H}$ II regions were reported by Moffat et al. (1979) with distances from $\approx 8$ to $2 \mathrm{kpc}$, which are not included in our data sample to keep data homogeneity. They are a continuation of young star groups associated to the spiral features revealed by the present analysis (see Fig. 3 in Moffat et al. 1979 and compare it to our Figs. 3 and 4 ). From $175^{\circ}$ to $185^{\circ}$, at $\sim 1.5 \mathrm{kpc}$ from the Sun, F68 shows a region labeled $\mathrm{L}$ with a reddening of up to $0.7 \mathrm{mag}$, in complete agreement with the reddening value we found for the cluster
NGC 2129. Apart from this object, we do not have young clusters at this location, but other, less young clusters show comparable reddening values. At the side $255^{\circ}-280^{\circ}$ along the plane, another dense cloud, labeled I in F68, precludes us from seeing far objects. In fact, Figures 5-8 in F68 show that the absorption starts increasing strongly beyond $0.5 \mathrm{kpc}$ from the Sun in this part of the TGQ.

From $215^{\circ}$ to $255^{\circ}$ (the Fitzgerald window) the F68 maps show that the reddening scarcely reaches values above 0.6 mag; in the mean, reddening goes from 0.1 to 0.6 mag and only close to the borders of the Fitzgerald window can values be found exceeding $0.6 \mathrm{mag}$. This fact is entirely confirmed by Figure 1 (top left panel), where with few exceptions, most of the clusters keep reddening values below 0.8 . Higher values as those at $230^{\circ}-240^{\circ}$ in the same figure may be due to the presence of other absorption regions, labeled $\mathrm{X}$ and $\mathrm{T}$ in Figure 4 of F68, located at $\sim 1$ and $2 \mathrm{kpc}$. Leaving these exceptions aside, the Fitzgerald window extension in both longitude and latitude is not only confirmed by our sample of open clusters, but we are in the position to confirm that it extends up to $12 \mathrm{kpc}$ from the Sun.

The top right panel in Figure $1\left[E_{(B-V)}\right.$ vs. Galactic latitude] shows the trend of all our objects to keep below the Galactic plane (negative latitudes). In particular, the bulk of young clusters and BPs is located mostly below the plane (with a peak at $b \approx-1^{\circ}$ ) extending down the plane, reaching $b \approx-15^{\circ}$. Just one nearby young object has been found at latitude $+4^{\circ}$. In the bottom right panel of Figure 1, the clusters/BPs exhibit an interesting distribution. Some of them are confined to a narrow band along $b \approx 0^{\circ}$ and can be found even at $8 \mathrm{kpc}$ from the Sun. Others that appear below the Galactic plane $\left(b<-1^{\circ}\right)$ are nearby or are all at more than $4 \mathrm{kpc}$, reaching distances as large as $12 \mathrm{kpc}$. However, comparing all four panels we conclude, from an optical point of view, that nearby young clusters are mostly found at $b<5^{\circ}$, showing a clear trend to be located below the formal Galactic 


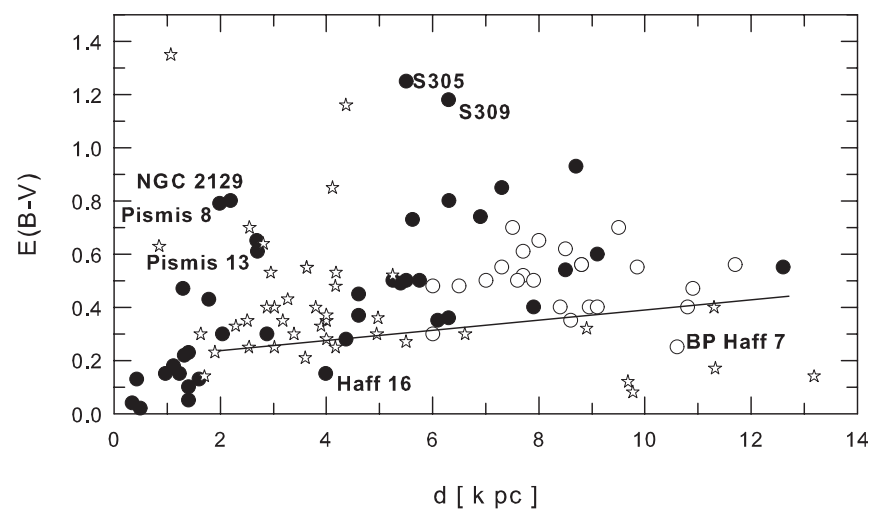

FIG. 2.-Young and old open clusters and BPs in the distance- $E_{(B-V)}$ plane. Symbols are as in Fig. 1. The solid line approximately represents the way the minimum reddening increases with distance.

plane $\left(b=0^{\circ}\right)$. More distant young clusters and some BPs take place along $b=0^{\circ}$ up to $\approx 8 \mathrm{kpc}$ from the Sun, although a few of them are found below the plane too. Nevertheless, the remarkable feature longward 6-7 kpc is the presence of BPs that, in the mean, are all in the range $-10^{\circ}<b<-2.5^{\circ}$, demonstrating the bending/widening of the distant Galactic plane at a distance of $\approx 8 \mathrm{kpc}$. The few young clusters in the bottom left panel clusters located as far as 9-12 kpc also follow the pattern defined by BPs. The reddening latitude distribution found with our clusters also confirms the findings of Figures 5-8 in F68 in the sense that most of the reddening happens in the solar neighborhood and that, below the plane, the reddening decreases quickly as the distance increases.

The overall confirmation of the way reddening is affecting our sample comes from Figure 2, where the reddening of each cluster is plotted against the distance to the Sun. Here we find conclusive evidence that most of the reddening in the TGQ takes place in the first kiloparsecs from the Sun. We interpret the pattern of Figure 2 as due to quick and large variations of dust concentration with longitude. In fact, extreme reddening values take place rapidly in the first $3 \mathrm{kpc}$ from the Sun. Three nearby young clusters show the largest reddening, Pismis 8 and 13 toward Vela and NGC 2129 at the beginning of the TGQ. There is then a paucity of objects, already noted above, which extends up to $4 \mathrm{kpc}$. Thereafter, the minimum of the reddening of young objects slowly increases with distance. However, the exceptions are two objects, one at $4 \mathrm{kpc}$ and the other at more than $10 \mathrm{kpc}$ (Haffner 16 and the BP Haff 7, respectively), that show reddening values similar to the ones found up to $1.5 \mathrm{kpc}$ from the Sun. The four old clusters at $d \geq 9.5 \mathrm{kpc}$ that show very low reddening values are all at $|b|>$ $7^{\circ}$. Very high reddening values are also found for distances from 4 to $7 \mathrm{kpc}$. A couple of young objects shown in Figure 2 at $5.5-6.5 \mathrm{kpc}(\mathrm{S} 305$ and $\mathrm{S} 309)$ and $l \approx 235^{\circ}$ have the highest values in our sample comparable to the reddening shown by two old, very reddened clusters at more than $4 \mathrm{kpc}$, which are located along the Galactic plane at $245^{\circ}<l<257^{\circ}$.

As a final comment, we see in Figure 2 that some very distant clusters, at more than $6 \mathrm{kpc}$, show minimum reddening values increasing slowly and maximum reddening values that strongly increase in the solar vicinity. This pattern has already been revealed by F68. That is, Figure 2 is indeed the prolongation in distance and sum of effects shown in panels $46-59$ of F68, where panel 51 is at the beginning of the Fitzgerald window and panel 56 is at the end of it. His panel 54, centered in the middle of the absorption window, shows the farthest objects at almost $9 \mathrm{kpc}$ from the Sun; we could extend our detections up to $12 \mathrm{kpc}$.

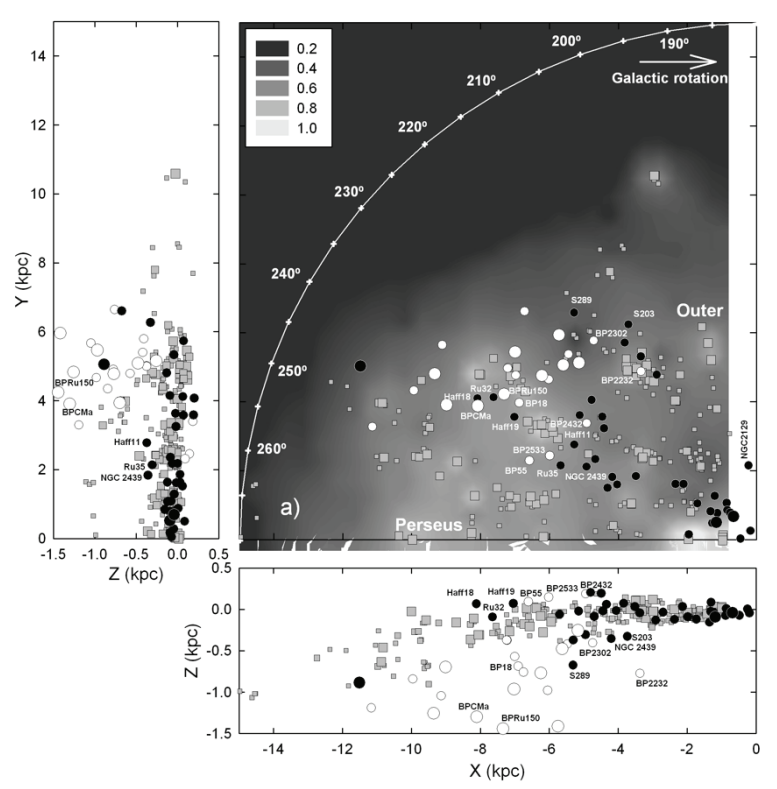

FIG. 3.- $\mathrm{CO}$ molecular cloud mass covering the mass ranges $0<M_{\odot} \leq 0.5$ (small gray squares), $0.5<M_{\odot} \leq 1$ (medium gray squares), and $>1 M_{\odot}$ (large gray squares), in units of $10^{5} M_{\odot}$ ) smoothing onto the $X$-Y plane from May et al. $(1988,1997,2007)$ data. For the smoothing process we use the nearest neighbors procedure and a $0.5 \mathrm{kpc}$ kernel. The inset shows the mass scale of the smoothing in units of $10^{5} M_{\odot}$. A scale of Galactic longitudes and the Galactic rotation are also shown. Some clusters and BPs ( filled and open circles, respectively) are indicated regarding their positions from the formal Galactic plane at $b=0^{\circ}$. The bottom and left panels show the $X-Z$ and $Y-Z$ projections, respectively, of molecular clouds, clusters, and BPs. No CO smoothing was performed in these panels. The Sun is at $(0,0)$. Large symbols denote the new fields observed, listed in Table 1.

Therefore, our assumption that most of the reddening occurs in the first kiloparsecs away the Sun but with a minimum value that keeps constant with distance is a full confirmation of the early findings of F68.

\section{THE THIRD GALACTIC QUADRANT: SPIRAL STRUCTURE}

Before entering in the details of the proposed spiral structure scenario, a general comment is in order. It is important to be aware of the uncertainties in the $\mathrm{CO}$ cloud kinematic distance determination in the TGQ. Brand \& Blitz (1993) show that there are important deviations from circular rotation in this quadrant. The velocity residuals that reflect the presence of noncircular motion can be as high as $40 \mathrm{~km} \mathrm{~s}^{-1}$ at around $240^{\circ}$ in longitude. Since circular rotation was assumed for all objects when fitting the rotation curve, the large velocity residuals found by Brand $\&$ Blitz indicate the presence of noncircular motions. Therefore, the kinematic distances obtained in the TGQ using their rotation curve might be subjected to large uncertainties, especially at those longitudes, like $240^{\circ}$, where the velocity residuals are important. For example, at $l=240^{\circ}$, deviations up to $20 \mathrm{~km} \mathrm{~s}^{-1}$ from the circular motion can produce uncertainties in the kinematic distances up to at least $50 \%$.

We show in Figure 3 (central panel) the mass density map of CO clouds from May et al. $(1997,2007)$ in the $X-Y$ plane after a smoothing process using the nearest neighbor method and a $0.5 \mathrm{kpc}$ kernel. Superposed on this density map we show the individual CO clouds (gray filled squares), the young open clusters (black filled circles), and BPs (open circles). All these stars have ages less than $100 \mathrm{Myr}$ (listed in Table 1) and are thus supposed to lie not far from their birthplaces, mostly in spiral arms. 


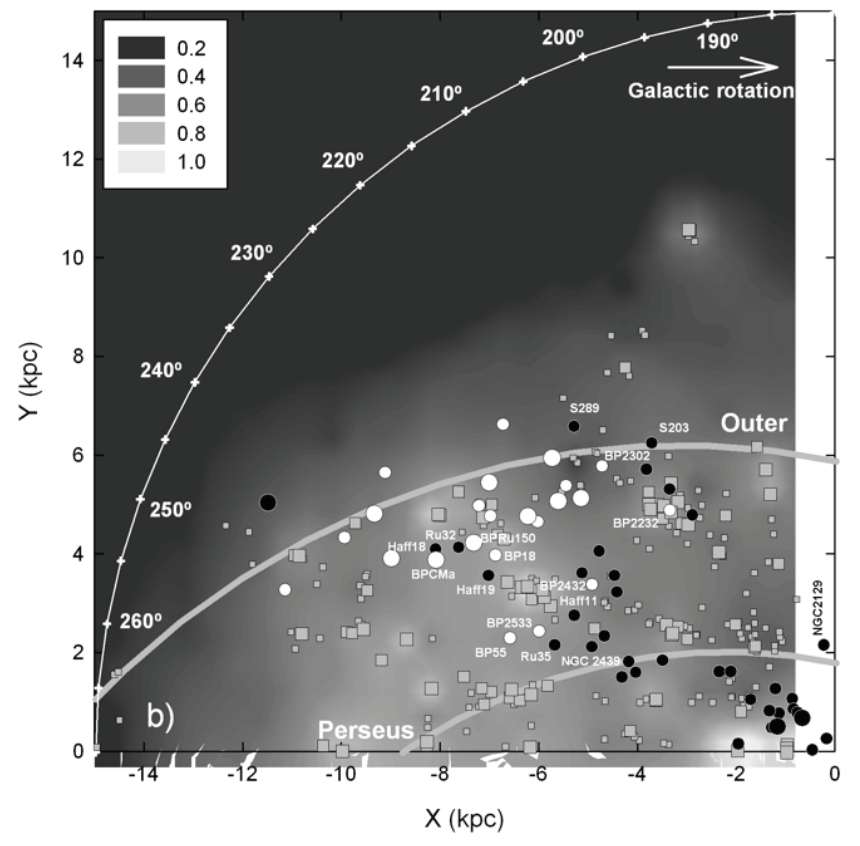

FIG. 4.- Same as Fig. 3, but including the Vallée (2005) arms.

The new analyzed fields are shown with slightly large symbols. The inset shows the CO mass scale of the figure in units of $10^{5} M_{\odot}$. The $X-Z$ (bottom panel) and the $Y$-Z (left panel) projections of these components are also shown. In these side panels, the density map is not shown since the smoothing process gives unreal contours in the $X-Z$ and $Y-Z$ projections due to the small $Z$ range and the large $X$ and $Y$ ranges. For a better understanding of the figure we have provided a longitude scale.

The $X-Y$ projection demonstrates that the $\mathrm{CO}$ is distributed throughout the whole TGQ showing a large mass concentration at $l \simeq 240^{\circ}$ and $\approx 8 \mathrm{kpc}$ from the Sun. Two other prominent concentrations of $\mathrm{CO}$ complexes are evident, one at $205^{\circ}<l<$ $215^{\circ}(-4<X<-1,4.5<Y<6)$ and the other at $255^{\circ}<l<$ $270^{\circ}(-6<X<-10,0<Y<3)$. We remark the extreme CO concentration nearby the Sun toward Vela $\left(l \simeq 260^{\circ}\right)$, which is the Vela Molecular Ridge (May et al. 1988).

In the map of Figure 4 (which is identical to Fig. 3) we have superposed the logarithm spiral arm models of Vallée (2005) that represent the grand design features expected in the TGQ.

Just to illustrate the uncertainty involved in trying to fit largescale spiral features to our Galaxy, we want to mention that the Russeil (2003) model for grand design features places the outer arm slightly closer and the Perseus arm slightly farther out.

\subsection{The Outer Arm}

From both optical and $\mathrm{CO}$ observations, the outer (Cygnus) arm in the TGQ clearly looks like a grand design spiral feature extending from $l=190^{\circ}$ to $255^{\circ}$, confirming the findings in Carraro et al. (2005) and Moitinho et al. (2006) as shown in Figure 4. Apart from not having optical tracers before $l=210^{\circ}$, both distributions (CO and stars) are similar in shape and extension and are spatially coincident. Furthermore, the data are well described by the Vallée (2005) model of the Cygnus arm, although a slight tendency to deviate from the logspiral model (being the $\mathrm{CO}$ and optical data at a slightly smaller distance from the Sun) is evident. This is part of the uncertainty in fitting the grand design features, as already noted above. The Russeil (2003) models would better fit this arm than the Vallée (2005) models, but in such a case other features in our scheme (e.g., the Perseus arm) will not fit at all. Such a shearing of the external parts of arms is often seen in other galaxies and is included in more sophisticated models of the Milky Way (Drimmel \& Spergel 2001).

The outer arm, as traced by our sample, coincides as well with the H I arm (Levine et al. 2006), and by extrapolating its extension to the fourth quadrant it appears to connect to the distant $\mathrm{H}$ I arm recently discovered by McClure-Griffiths et al. (2004). At $l=210^{\circ}$ we find components of this arm at the height of the formal Galactic plane, $b=0^{\circ}$, but also others (S283 and S289) that are well below the plane. At longitudes greater than $l=210^{\circ}$ the outer arm is basically defined by BP stars. The left panel of Figure 3 shows the way that this arm starts to vertically develop and bend. The distance from the Sun of this structure goes from $\simeq 7 \mathrm{kpc}$ at $l=210^{\circ}$ to $12 \mathrm{kpc}$ at $l=255^{\circ}$, having a varying width of $2-3 \mathrm{kpc}$. The lower panel, the $X-Z$ projection, shows how the outer arm is warped. In summary, this arm enters the TGQ at $b=0^{\circ}$ and stays more or less coincident with the formal Galactic plane up to $l=210^{\circ}$, where it starts bending, reaching its maximum height $Z$ about $1.3-1.5 \mathrm{kpc}$ below the $b=0^{\circ}$ plane around $l=250^{\circ}$. The arm has a sizable vertical width of over half a kiloparsec.

\subsection{The Local Arm}

The Local (Orion) arm has been traced with some detail in the first Galactic quadrant, but it is not very well known in the TGQ. Historically, the situation is somewhat confusing, with some studies placing it toward $l \simeq 240^{\circ}$ and others at $l \simeq 260^{\circ}$. As we see in this section, the source of confusion is that there appear to be actually two distinct structures in the solar vicinity. Moreover, the adoption of either direction as "the" Local arm is a result of the particular samples used in each of the previous studies. This is especially evident when one considers that studies relied mostly on either optical or radio observations.

In the optical, Moffat et al. (1979) presented evidence that this arm is a well-confined structure located at $l=244^{\circ}$ toward Canis Major, while Vega et al. (1986) situate it at $l=260^{\circ}$ toward Vela. Our optical data clearly show that a bridge exists between the Sun and the outer arm at $l=245^{\circ}$ toward Canis Major. In other words, we see young stars clusters and BPs at any distance between the Sun and the outer arm in this direction (Moitinho et al. 2006) covering from $l=230^{\circ}$ to $250^{\circ}$, due to the particular location of the Sun. Furthermore, there are CO cloud complexes distributed in a similar way. The same happens in Figures 3 and 4, which show how the distribution of the clusters/BPs and CO clouds of our sample is compatible with this structure being the extension of the Local arm into the TGQ. Hereafter, we refer to this structure as the Local arm.

We warn the reader, however, that the situation is more complicated as far as CO clouds are concerned. The Perseus arm in the second quadrant exhibits quite significant streaming motion. With the data at disposal we cannot exclude that $\mathrm{CO}$ clouds in this particular region of the TGQ $\left(240^{\circ}-245^{\circ}\right)$ may be part of the Perseus arm and their diffused structures simply due to streaming motions.

The Local arm extends then up to $d \approx 8 \mathrm{kpc}$ and has its barycenter at $l \approx 245^{\circ}$. The optical observations indicate that clusters in the Local arm stay close to, or below, the $b=0^{\circ}$ plane in the solar neighborhood. Some few BPs follow this trend too, and three clusters (Haffner 11, Ruprecht 35, and NGC 2439) are clearly well below the Galactic plane. For $d \geq 8 \mathrm{kpc}$ from the Sun, the vertical extension of the Local arm (see BPs in Fig. 3) increases with the distance to the Sun and reaches the outer arm at the point where it seems to show the maximum optical warping. 
Thus, the Local arm appears as an interarm feature, a bridge from the Sun up to the outer arm resembling similar structures very common in face-on spiral galaxies (see, e.g., the galaxy M74 picture in $\S 5.3$ ). We stress that the extension and shape of the distributions of the young stellar component and CO complexes, together with their spatial coincidence, fulfill the basic requirements for the presence of a spiral arm.

For $l \geq 250^{\circ}$ no stellar groups are found in our sample except for a few nearby clusters. This lack of distant tracers is likely due to the visual obstruction caused by the Gum Nebula $(l=$ $258^{\circ}, b=-2^{\circ}$ ) at $0.5 \mathrm{kpc}$ from the Sun covering $\approx 36^{\circ}$ in the sky. The IRAS Vela shell is located in front of it (Woermann et al. 2001), while behind the Gum nebula extend the Vela association and the very massive CO structures that compose the Vela Molecular Ridge (May et al. 1988).

However, and despite not having optical tracers at large heliocentric distances for $l \geq 250^{\circ}$, the $\mathrm{CO}$ emission still continues and is present at several distances in the Vela direction with a noticeable concentration of massive clouds reaching $2-3 \mathrm{kpc}$ from the Sun (the Vela Molecular Ridge) followed by a sparse series of other CO complexes reaching $12 \mathrm{kpc}$. In particular, we call attention to the massive complexes at $-8<X<-10,1<$ $Y<3$. From a radio point of view, the Vela molecular ridge and the $\mathrm{CO}$ complexes beyond it exhibit the emission continuity and physical extension required to conform a spiral feature and have thus also been interpreted as the extension of the Local arm in the TGQ. In this view, the Sun is inside the "Local arm," which is defined by $\mathrm{CO}$ alone in the range $250^{\circ} \leq l \leq 270^{\circ}$ (except for possibly $2-3$ clusters within $2 \mathrm{kpc}$ ) and bends toward $l=260^{\circ}$.

Besides the sample biases mentioned in the beginning of this section, the conclusions of early studies were even more affected by their limited depth, which only allowed one to explore with some detail the first 3-4 kpc from the Sun. From our new map presented in Figures 3 and 4, it is seen how that is not deep enough. Indeed, the Vela Molecular Ridge, which seemed to indicate the presence of a massive structure extending toward $l=260^{\circ}$, does not reach beyond $2-3 \mathrm{kpc}$, and although there are farther complexes more or less in the same direction, they are sparse and some are even likely part of the Perseus arm (see $\S 5.3$ ). This is in contrast with the structure seen toward $l=240^{\circ}$, which is much better delineated as well as also being defined by young stellar populations.

As a further remark, Alfaro et al. (1991), using open clusters, pointed out the existence of a depression at $l=240^{\circ}$, which they called the Big Dent, an elliptical structure about $2-3 \mathrm{kpc}$ wide, extending down to 200 pc below the Galactic plane at a distance of $2 \mathrm{kpc}$ from the Sun. This depression has been confirmed in Moitinho (2002) and can also be identified in the $Z-X$ projection of the bottom panel of Figure 2 of Moitinho et al. (2006) and Figure 3 of this paper. According to our scenario, this structure is part of the Local arm. It is interesting to note that the depression, together with the star-forming complex found near its deeper part (Moitinho 2002), fit well the description of a corrugated arm with star-forming complexes in its peaks and valleys (e.g., Carina-Sagittarius; Alfaro et al. 1992).

\subsection{The Perseus Arm}

This arm is well known in the second Galactic quadrant $(\mathrm{Xu}$ et al. 2006), but its existence has not been clearly established in the TGQ after $l=190^{\circ}$. NGC 2129 (close to the anticenter) is a possible member of Perseus, but the rest of the optical data do not show much evidence of the arm. On the stellar side, only a clump of clusters at $6 \mathrm{kpc}$ at $l \approx 245^{\circ}$ would be consistent with belonging to the arm. However, they also nicely fit the much

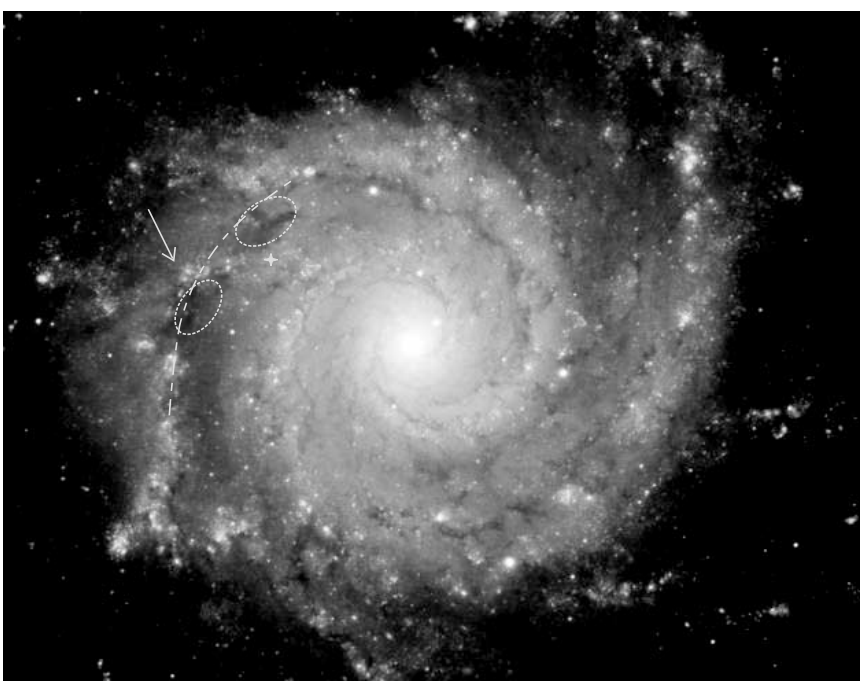

FIG. 5.-Face-on galaxy M74. As indicated in $\S 5.3$, the top left corner sketches (long-dashed line) a grand design spiral arm (like Perseus in the MW) defined by dust tracers (dashed ellipses), together with the lack of stellar counterparts. This arm is crossed by an interarm spiral feature that extends to the outer galaxy (like the Local arm in the MW). This happens thanks to the presence of an absorption window in between the two ellipses. We show a tentative location for the Sun (star) and the crossing region that contains an enhanced number of young star clusters (arrow). [See the electronic edition of the Journal for a color version of this figure.]

more obvious structure defining the Local arm. It is thus unclear whether the clump belongs to the Local arm or to Perseus.

The situation changes when one considers the $\mathrm{CO}$ observations. Complexes appear at $l \approx 260^{\circ}(-6<X<-8,0<Y<1.5)$ and $l \approx 215^{\circ}(-1<X<-2,2<Y<3)$, which could be defining Perseus. The complex at $l \approx 235^{\circ}(-3<X<-4,2<$ $Y<2.5)$ could also belong to Perseus, although it is displaced about $0.5 \mathrm{kpc}$ toward the anticenter relative to the Vallée (2005) curve.

The presence of dust tracers together with the lack of a stellar counterpart in certain regions of grand design arms is not unexpected. An excellent example is the upper left of the M74 galaxy shown in Figure 5. ${ }^{5}$ Interestingly, the resemblance of Perseus with the arm in M74 does not ends here. In fact, the region of interest in M74 mimics our proposed scenario for the TGQ: A grand design arm that is only defined by dust over a large extent (like Perseus) is crossed by an interarm spiral feature that extends to the outer galaxy (like the Local arm). Moreover, the crossing region contains an enhanced number of young star clusters (as the clump discussed above). Our data seem therefore to indicate that the crossing of the Local arm is disrupting Perseus in the TGQ. This could also explain the displacement of the CO complex at $l \approx 235^{\circ}$.

\section{THE SIGNATURE OF THE WARP}

Figure 6 shows, from top to bottom, the CO complexes and clusters/BPs in the $l, Z$ plane distributed according to increasing distances from the Galactic center. This figure provides a strong demonstration of the Galactic stellar and molecular warp. In the top panel, most clusters, BPs, and CO clouds are all confined to the formal plane $\left(b=0^{\circ}\right)$ with a slight trend to increasing the vertical dispersion for approximately $l \geq 230^{\circ}$. The exception is the BP in the field of NGC 2232. In the middle panel we can see a huge vertical development of the Galactic disk with different components: $\mathrm{CO}$ clouds and clusters compose a thin disk with a

\footnotetext{
5 See M74 at http://apod.nasa.gov/apod/ ap011004.html.
} 


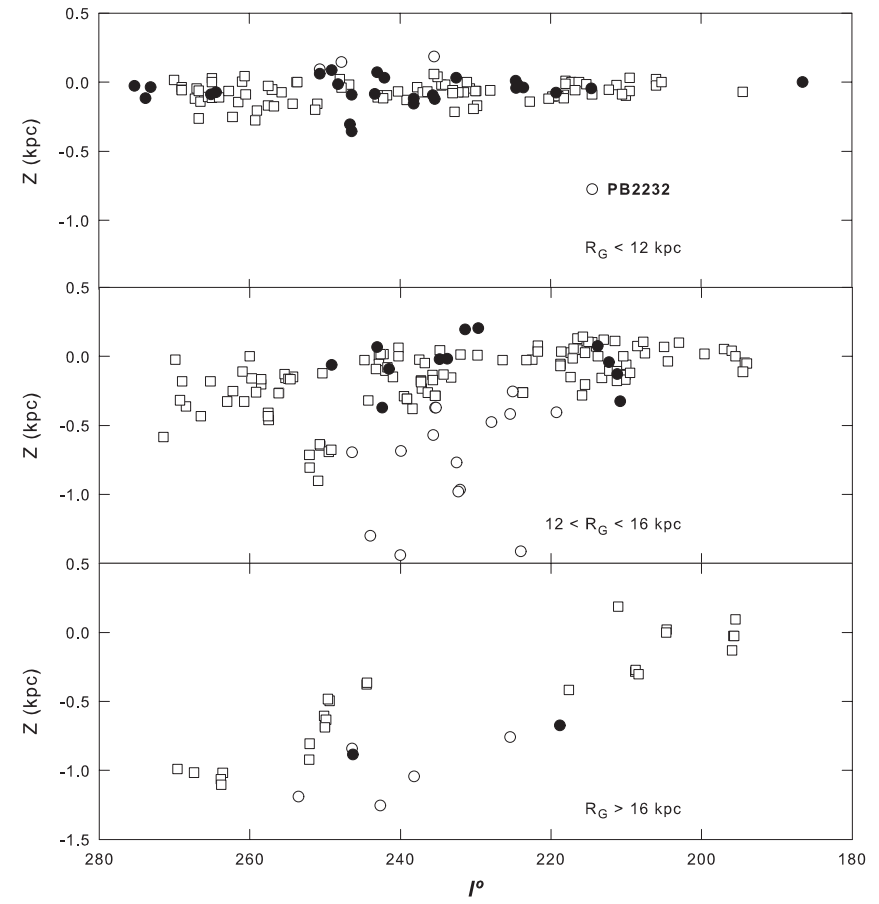

FIG. 6.- - Young open clusters ( filled circles), BPs (open circles), and CO clouds (open squares) in the $l-Z$ projection distributed according to the distance from the center of the Galaxy.

more defined trend to lie below the formal plane for $l \geq 240^{\circ}$; BPs start falling below the plane for $l \geq 220^{\circ}$ and a few clouds are found well below the plane at $l \geq 250^{\circ}$. The bottom panel is, indeed, striking: the most remote objects (be they molecular clouds, clusters, or BPs) all fall abruptly below the plane at $l \geq 210^{\circ}$. Although limited in longitude, this last panel suggests that the maximum stellar warp occurs at $l=240^{\circ}-245^{\circ}$, while the maximum of the molecular warp seems to take place at $l \geq 260^{\circ}$.

\section{FINAL REMARKS AND CONCLUSIONS}

We have provided a new detailed picture of the spiral structure of the Galactic disk in the third quadrant by combining together optical and $\mathrm{CO}$ observations. The most striking structures in this zone of the Galaxy are the outer (Cygnus) and Local (Orion) arms. The observed outer arm is well matched by the extrapolation of the Vallée (2005) spiral curve, and the Local arm extends toward $l \approx 245^{\circ}$ up to the limit of both optical and CO observations, reaching the outer arm and confirming our previous results
(Moitinho et al. 2006). The more thorough analysis presented in this paper has revealed previously overlooked evidence for the presence of Perseus in the TGQ. Unlike the outer and Local arms, which are defined by both CO clouds and young stellar populations, Perseus appears to be defined almost solely by CO. The lack of stellar tracers in Perseus is similar to what is observed in a grand design arm in M74. We hypothesize that the crossing of the Local arm is disrupting Perseus in the TGQ. It is worth remarking that the recent $\mathrm{H}$ I map (see Levine et al. 2006, Fig. 2) displays structures consistent with our description of the outer, Local, and Perseus arms.

We have addressed the historical discrepancy between optical and radio studies regarding the direction of the Local arm. We find that previous radio indications of the Local arm being directed toward $l \approx 260^{\circ}$ are heavily based on the presence of the Vela Molecular Ridge. This massive CO complex is confined, however, to $2-3 \mathrm{kpc}$ from the Sun. A few other CO clouds are also seen in the same general direction, but they are sparsely distributed and some are likely part of the Perseus arm.

As for the reddening distribution, we are in the position to generalize and confirm earlier findings by F68 in the sense that (1) most of the $E_{B-V}$ in the TGQ takes place in the first 2-3 kpc from the Sun, (2) thanks to the longitude extension of a window absorption we see very far objects, (3) objects in this window can be detected at more than $10 \mathrm{kpc}$ from the Sun, and (4) the $E_{B-V}$ in the Vela direction is simply huge, precluding optical observations of far objects behind it.

Finally, we have found clear signatures of the warp in the TGQ, which reaches at least $1 \mathrm{kpc}$ below the $b=0^{\circ}$ plane. The maximum stellar warp occurs at $l=240^{\circ}-245^{\circ}$, while the maximum of the molecular warp seems to take place at a larger longitude, $l \geq 260^{\circ}$. Whether the maximum of the stellar warp is displaced (ahead) with respect to that of the gaseous warp requires an analysis of the fourth quadrant. This study is now underway.

R. A. V. and G. L. B. wish to acknowledge financial support from the IALP (UNLP-CONICET, Argentina) and PIP 5970 from CONICET. J. M. and L. B. acknowledge support from the Chilean Center for Astrophysics FONDAP 15010003. The work of G. C. was supported by a contract from the Departamento de Astronomía, Universidad de Chile. A. M. acknowledges support from FCT (Portugal) through grant POCI/CTE-AST/57128/2004. We wish to thank the anonymous referee for valuable comments and suggestions that greatly contributed to improve this article.
Abt, H. A. 1979, ApJ, 230, 485

Alfaro, E. J., Cabrera-Cano, J., \& Delgado, A. J. 1991, ApJ, 378, 106 . 1992, ApJ, 399, 576

Baume, G., Vázquez, R. A., \& Feinstein, A. 1999, A\&AS, 137, 233

Bellazzini, M., Ibata, R., Monaco, L., Martin, N., Irwin, M. J., \& Lewis, G. F. 2004, MNRAS, 354, 1263

Brand, J., \& Blitz, L. 1993, A\&A, 275, 67

Bronfman, L., Cohen, R. S., Alvarez, H., May, J., \& Thaddeus, P. 1988, ApJ, 324,248

Burton, W. B. 1985, A\&AS, 62, 365

Carney, B. W., \& Seitzer, P. 1993, AJ, 105, 2127

Carraro, G., Chaboyer, B., \& Perencevich, J. 2006, MNRAS, 365, 867

Carraro, G., Moitinho, A., Zoccali, M., Vázquez, R. A., \& Baume, G. 2007, AJ, 133,1058

Carraro, G., Vázquez, R. A., Moitinho, A., \& Baume, G. 2005, ApJ, 630, L153

Cohen, R. S. 1983, in Surveys of the Southern Galaxy, ed. W. B. Burton \& F. P. Israel (Dordrecht: Reidel), 265

Dean, J. F., Warren, P. R., \& Cousins, A. W. J. 1978, MNRAS, 183, 569

Drimmel, R., \& Spergel, D. N. 2001, ApJ, 556, 181

\section{REFERENCES}

Fitzgerald, M. P. 1968, AJ, 73, 983

Forbes, D. 1996, AJ, 112, 1073

Giorgi, E. E., Baume, G., Solivella, G., \& Vázquez, R. A. 2005, A\&A, 432, 491

Girardi, L., Bressan, A., Bertelli, G., \& Chiosi, C. 2000, A\&AS, 141, 371

Landolt, A. U. 1992, AJ, 104, 340

Levine, E. S., Blitz, L., \& Heiles, C. 2006, Science, 312, 1773

Martin, N. F., Ibata, R. A., Bellazzini, M., Irwin, M. J., Lewis, G. F., \& Dehnen, W. 2004, MNRAS, 348, 12

Martínez-Delgado, D., Butler, D. J., Rix, H.-W., Franco, Y. I., Peñarrubia, J., Alfaro, E. J., \& Dinescu, D. I. 2005, ApJ, 633, 205

May, J., Alvarez, H., \& Bronfman, L. 1997, A\&A, 327, 325

May, J., Bronfman, L., Max-Moerbeck, W., \& Olmos, F. 2007, ApJ, submitted May, J., Murphy, D. C., \& Thaddeus, P. 1988, A\&AS, 73, 51

McClure-Griffiths, N. M., Dickey, J. M., Gaensler, B. M., \& Green, A. J. 2004, ApJ, 607, L127

Meynet, G., Mermilliod, J.-C., \& Maeder, A. 1993, A\&AS, 98, 477

Moffat, A. F. J., Jackson, P. D., \& Fitzgerald, M. P. 1979, A\&AS, 38, 197

Moitinho, A. 2001, A\&A, 370, 436 
Moitinho, A. 2002, in ASP Conf. Ser. 285, Modes of Star Formation and the Origin of Field Populations, ed. E. K. Grebel \& W. Brandner (San Francisco: ASP), 256

Moitinho, A., Alfaro, E. J., Yun, J. L., \& Phelps, R. L. 1997, AJ, 113, 1359

Moitinho, A., Vázquez, R. A., Carraro, G., Baume, G., Giorgi, E. E., \& Lyra, W. 2006, MNRAS, 368, L77

Momany, Y., Zaggia, S., Bonifacio, P., Piotto, G., De Angeli, F., Bedin, L. R., \& Carraro, G., 2004, A\&A, 421, L29

Momany, Y., Zaggia, S., Gilmore, G., Piotto, G., Carraro, G., Bedin, L. R., \& de Angeli, F. 2006, A\&A, 451, 515

Murphy, D. C., \& May, J. 1991, A\&A, 247, 202

Ogawa, H., Mizuno, A., Hoko, H., Ishikawa, H., \& Fukui, Y. 1991, Int. J. Infrared Millimeter Waves, 11, 717
Schmidt-Kaler, T. 1982, in Landolt-Börnstein, Numerical Data and Functional Relationship in Science and Technology, ed. K. Schaifers \& H. H. Voigt (Berlin: Springer), 15

Stetson, P. B. 1987, PASP, 99, 181

Turner, D. G., Grieve, G. R., Herbst, W., \& Harris, W. E. 1980, AJ, 85, 1193 Vallée, J. P. 2005, AJ, 130, 569

Vega, E. I., Muzzio, J. C., \& Feinstein, A. 1986, Rev. Mex. AA, 13, 33

Woermann, B., Gaylard, M. J., \& Otrupcek, R. 2001, MNRAS, 325, 1213

Wouterloot, J. G. A., Brand, J., Burton, W. B., \& Kwee, K. K. 1990, A\&A, 230,21

Xu, Y., Reid, M. J., Zheng, X. W., \& Menten, K. M. 2006, Science, 311, 54 